\title{
Empirically driven orthonormal bases for functional data analysis
}

\author{
Nassar, Hiba; Podgorski , Krzysztof
}

Published in:

Numerical Mathematics and Advanced Applications ENUMATH 2019

Link to article, DOI:

10.1007/978-3-030-55874-1_76

Publication date:

2021

Document Version

Peer reviewed version

Link back to DTU Orbit

Citation (APA):

Nassar, H., \& Podgorski , K. (2021). Empirically driven orthonormal bases for functional data analysis. In F. J. Vermolen, \& O. Vuik (Eds.), Numerical Mathematics and Advanced Applications ENUMATH 2019 Springer. Lecture Notes in Computational Science and Engineering https://doi.org/10.1007/978-3-030-55874-1_76

\section{General rights}

Copyright and moral rights for the publications made accessible in the public portal are retained by the authors and/or other copyright owners and it is a condition of accessing publications that users recognise and abide by the legal requirements associated with these rights.

- Users may download and print one copy of any publication from the public portal for the purpose of private study or research.

- You may not further distribute the material or use it for any profit-making activity or commercial gain

- You may freely distribute the URL identifying the publication in the public portal

If you believe that this document breaches copyright please contact us providing details, and we will remove access to the work immediately and investigate your claim 


\title{
Empirically driven orthonormal bases for functional data analysis
}

\author{
Hiba Nassar and Krzysztof Podgórski
}

\begin{abstract}
In implementations of the functional data methods, the effect of the initial choice of an orthonormal basis has not been properly studied. Typically, several standard bases such as Fourier, wavelets, splines, etc. are considered to transform observed functional data and a choice is made without any formal criteria indicating which of the bases is preferable for the initial transformation of the data. In an attempt to address this issue, we propose a strictly data-driven method of orthonormal basis selection. The method uses $B$-splines and utilizes recently introduced efficient orthornormal bases called the splinets. The algorithm learns from the data in the machine learning style to efficiently place knots. The optimality criterion is based on the average (per functional data point) mean square error and is utilized both in the learning algorithms and in comparison studies. The latter indicate efficiency that could be used to analyze responses to a complex physical system.
\end{abstract}

\section{Introduction}

Functional data analysis (FDA) is the field in statistics that studies the analysis and theory of data that are in the form of functions, images, and shapes, etc, see [3]. The FD may come as a dynamical response from a physical system subject to stochastic excitation that can be written in a generic form as

$$
H\left(y^{(n)}, \ldots, y^{\prime \prime}, y^{\prime}, y, x ; \theta\right)=F(t),
$$

where $F(t)$ is a realization of stochastic forcing of the system whose response is given by $H$ that involves some physical parameters given in $\theta$. Often the response from such a system is stochastic not only because of random excitation $F$ but also due to randomness in the parameter $\theta$ of the system. As a result, the responses $y_{i}(t)$ from such a system can be conceptually treated as FD depending both on $\theta_{i}$ and

Hiba Nassar

Department of Statistics, Lund University, Sweden. e-mail: hiba.nassar@stat.lu.se

Krzysztof Podgórski

Department of Statistics, Lund University, Sweden. e-mail: krzysztof.podgorski@ stat.lu.se 
$F_{i}(t)$. The goal is to obtain an efficient treatment of these functional observations in order to infer about $\theta$ as well as about the functional structure of $y$.

FD are not observed as continuous objects, but rather as discrete data. Highfrequency sampling and mathematical efficiency allow these data to be seen as samples of curves, surfaces or anything else varying over a continuum. The fundamental step in FDA is to convert this discrete recorded data to a functional form, which gives each function the possibility to be evaluated for all values of $t$. To utilize the topology of such data for the dimension reduction one performs the data conversion. One of the methods used is to represent a functional object as a linear combination of coefficients and a number of suitable basis functions. For the purpose, one of the standard bases such as trigonometric, wavelet, or polynomial is typically chosen.

The efficiency is accomplished by using smoothing through regression or roughness penalty for estimating the coefficients of the basis expansions. However, all such analyses are preceded by the initial choice of a functional basis used to analyze data, which is hardly objective and often driven by mathematical convenience. On the other hand, it is both theoretically and practically observed that the choice of the basis affects efficiency in retrieving the functional structure of a studied model. This motivated us to investigate this problem more thoroughly.

In the spirit of the main data analysis paradigm, for a given FD set it may be computationally effective to work with a data-driven basis. Consider, for example, the classical smoothing problem, where for a given data we want to fit a smooth function. Using the $B$-splines together with a regularization method, for example the Lasso method, one may selectively choose a subspace of the spline space by shrinking parameters to zero, see [4]. Such a basis can be chosen for each FD sample but a choice valid for all samples is not obvious.

We acknowledge the value of splines in FDA but we proceed differently by utilizing freedom in knots placement to algorithmically search for efficient knots patterns and utilize them in the orthonormal basis construction. In the process, we implement machine learning algorithms for the choice of basis reducing the mean square error (MSE) uniformly for all samples and study its efficiency against other choices of the basis. The optimality criterion is utilized, both in the learning algorithms and in comparison studies. This criterion allows for comparison performances of different bases in a given problem. After efficiently learning from the data about knot placements, we utilize the new construction of the orthonormal spline bases, termed splinets and introduced in [9]. There, it is demonstrated that the splinets are characterized by optimality properties that bring further benefits to our approach.

\section{FD and their representations}

Discrete observations of a single function $x(t), t \in[0,1]$, at times $t_{j}$ result in

$$
y_{j}=x\left(t_{j}\right)+\epsilon_{j}, \quad j=1, \ldots, p
$$

where $\epsilon_{j}$ is an error term in the data. To account on topological features assumed to be present in $x(t)$, the function is assumed to be smooth. One of the most common ways of representing it efficiently is by using the basis function expansion, i.e. by decomposing linearly the function $x(t)$ in terms of a chosen basis system $\phi_{k}(t)$ 
consisting of $K \leq \infty$ basis functions

$$
x(t)=\sum_{k=1}^{K} c_{k} \phi_{k}(t) .
$$

The most commonly used basis functions $\phi_{k}(t)$ are Fourier, polynomial, splines and wavelets. It is typically assumed that the observations $x_{k}(t)$ are random elements of $L^{2}[0,1]$. In this Hilbert space, we use inner product $\langle\cdot, \cdot\rangle$ for an integral of the product of its two functional elements and which generates the norm $\|\cdot\|$. We call $L^{2}$-valued data functional observations. We use upper case and lower case letters in the context of FD in a similar manner as in the classical statistical convention, i.e. $X$ is yet not observable random element, while $x=x(\cdot)$ stands for its particular observed functional realization, i.e. a functional outcome of random experiment carried out according to the probability model for $X$.

All random functions are assumed to be square integrable, i.e. $E\|X\|^{2}<\infty$. In this context, one have to point to a classical result, the Karhunen-Loève expansion, see [8], which shows that the basis associated with this expansion has the optimality in the average mean square error sense, for more details see [6]:

$$
X(t)=\sum_{k=0}^{\infty} \sqrt{\lambda_{k}} Z_{k} e_{k}(t),
$$

where $\lambda_{k}$ is a square summable sequence of non-negative numbers, $e_{k}, k \in \mathbb{N}_{0}$ is an orthonormal (non-random) basis in $L^{2}[0,1]$ and $Z_{k}$ is a sequence of zero-mean variance-one uncorrelated random variables. In the Gaussian case, $Z_{k}$ are independent standard normal variables. The desired optimality of the basis $\left(e_{k}\right)$ is mostly of theoretical value since except for Brownian motion $\left(\lambda_{k}=(\pi k-\pi / 2)^{-2}, e_{k}(t)=\right.$ $\sqrt{2} \sin ((\pi k-\pi / 2) t))$ and Brownian bridge $\left(\lambda_{k}=(\pi k)^{-2}, e_{k}(t)=\sqrt{2} \sin (\pi k t)\right)$, the actual form of the optimal basis is not available. It is the central problem of the FDA to find the approximation of $e_{k}$ for any specific problem. To do this one has to decompose the original data using some basis of convenience.

The most popular decomposition of a function is by the Fourier basis

$$
\{\sqrt{2} \sin (2 \pi n t) ; n \in \mathbb{N}\} \cup\{\sqrt{2} \cos (2 \pi n t) ; n \in \mathbb{N}\} \cup\{\mathbf{1}\} .
$$

Fourier basis functions form an orthogonal basis and have good computational properties. A Fourier series is especially useful for extremely stable functions where there are no strong local features and the same curvature order everywhere. However, they are improper for data where discontinuities in the function itself or in low order derivatives are known or suspected [11, page 48]. In Fig. 2, the graphs in the second column illustrate a 40-dimensional Fourier approximation of functional signals.

Spline functions are a natural choice for approximating non-periodic FD. Splines combine the fast computation of polynomials with substantially greater flexibility. We explain some essential background of the $B$-splines, for more details we refer the reader to standard texts such as $[2,12]$.

A $B$-spline is a smooth function that consists of polynomial pieces that have the same degree, connected smoothly at join points $\xi_{0}<\xi_{1}<\cdots<\xi_{n+1}$, referred to as 


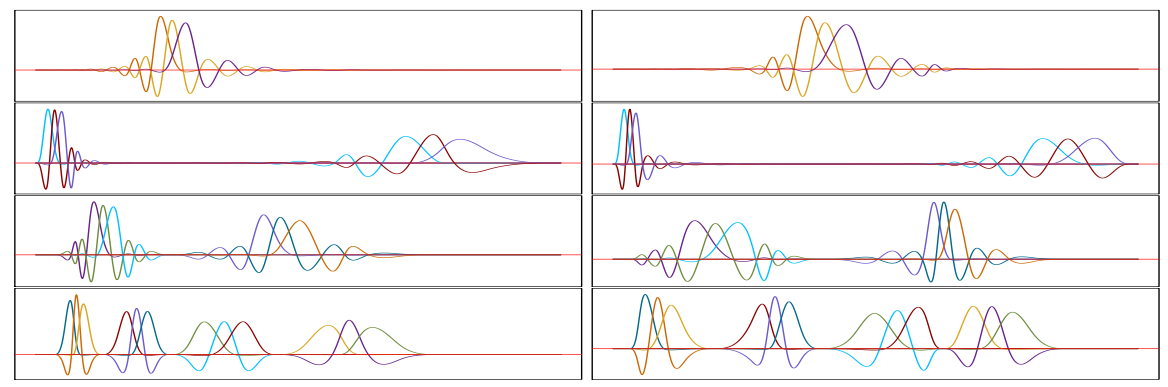

Fig. 1 Splinets for the data driven knots placement for two examples of Sect. 5

knots. The $B$-splines are sensitive to the choice of the knots' position, which is behind our main idea of the basis selection since the choice of the knots can be data-driven. Once the knots are set, $B$-splines can be effectively evaluated in a recursive way for any degree using the Cox-de Boor formula [2]. The $B$-splines have interesting properties that characterize it. Namely, all $B$-splines are positive, differentiable up to a certain level (the spline order) and have minimal compact intervals for their supports. But except for the case of order zero, the $B$-splines are not orthogonal. Different orthogonalization methods appeared in the literature but we are using our structured orthogonalization that creates basis systems for which we coined the term splinet. The splinet is prioritized over other orthonormal spline systems as it preserves locality and computational efficiencies of the original splines. For a more detailed explanation, we refer the reader to [9]. In Fig. 2, the graphs in the last column illustrate 40-dimensional, third-order $B$-spline decomposition of functional signals.

\section{Data driven choice of the knots}

The degree of a polynomial and the placement of knots defines the spline basis. We propose machine learning style techniques for the placement of the knots. The chosen knots are used to build splines basis functions $\phi_{k}(t)$ that are used in basis function expansion to convert the data from discrete recorded data into a functional one. The method of adding knots is based on the mean square error effectiveness of approximating the FD. The method is iterative and resembles the regression tree building by which it was inspired, see [5, Chapter 9].

For any FD set $\mathcal{X}=\left\{x_{i} \in L^{2}, i=1, \ldots n\right\}$, the set of best least square constant predictors is a set of functions

$$
x_{i}^{(0)}=\left\langle x_{i}, \mathbf{1}\right\rangle \mathbf{1}=\int x_{i} \cdot \mathbf{1} .
$$

The constant functions over the entire domain $[0,1]$ can be viewed as 0 -order splines with no internal knot points, and its one dimensional basis is given by the constant function 1. We set the initial set of knots to an empty set, i.e. $\mathcal{K}^{(0)}=\emptyset$, the initial basis $\mathcal{B}^{(0)}=\{\mathbf{1}\}$, and the projection to the space spanned by $\mathcal{B}^{(0)}$ is given by $\mathbf{P}^{(0)} x=$ $\langle x, \mathbf{1}\rangle \mathbf{1}$. The average mean square error (AMSE) per function of the approximations of $x_{i}$ 's by the optimal constant functions is given by 

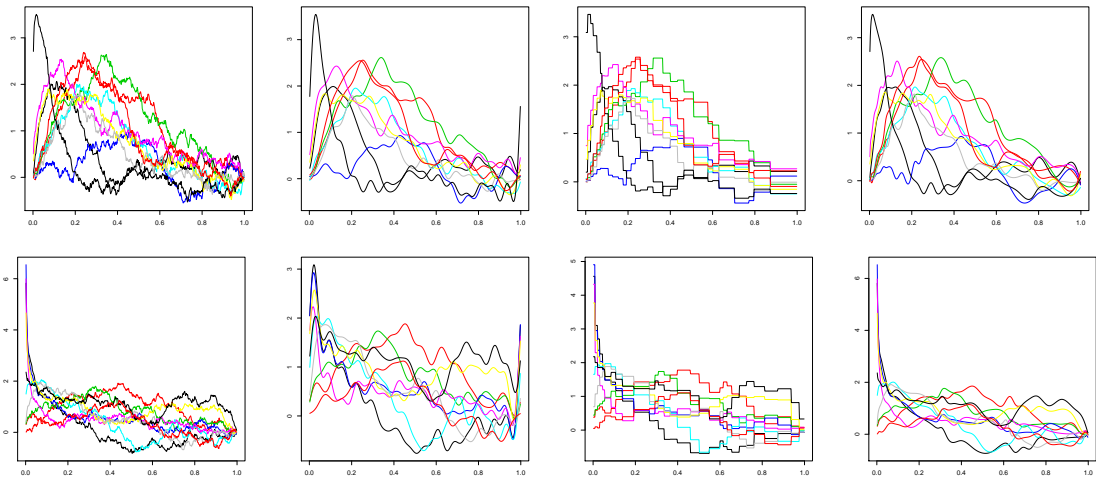

Fig. 2 Left: Ten samples of FD obtained from two different random functional of Brownian bridge. Middle-Left: Fourier approximations of the FD based on 30 Fourier basis functions. Middle-Right: Piecewise constant orthonormal basis approximations of the FD based on 30 basis functions. Right: Smooth splinet basis approximations of the FD based on 30 basis functions.

$$
\operatorname{AMSE}\left(\mathcal{Y}, \mathcal{B}^{(0)}\right)=\frac{1}{n} \sum_{i=1}^{n}\left\|x_{i}-\mathbf{P}^{(0)} x_{i}\right\|^{2}=\frac{1}{n} \sum_{i=1}^{n}\left\|x_{i}-\left\langle x_{i}, \mathbf{1}\right\rangle \mathbf{1}\right\|^{2} .
$$

The method at the first step, $s=1$, finds a knot $\xi \in[0,1]$ such that the optimal approximation of $x$ by a linear combination of the 0 -order splines with the set of knots $\mathcal{K}^{(1)}=\mathcal{K}^{(0)} \cup\{\xi\}$ yields the smallest AMSE between the FD $x_{i}$. In other words, denote by $\mathcal{B}^{(1)}(\xi)$ the orthonormal basis of piecewise constant functions over the intervals given by the knots in $\mathcal{K}^{(1)}(\xi)$. The new knot $\xi_{\text {new }}$ is chosen as

$$
\xi_{\text {new }}=\underset{\xi \in(0,1]}{\operatorname{argmin}} \operatorname{AMSE}\left(\mathcal{Y}, \mathcal{B}^{(s)}(\xi)\right) .
$$

Then the new, enlarged by one function, basis $\mathcal{B}^{(1)}=\mathcal{B}^{(1)}\left(\xi_{\text {new }}\right)$ is uniquely defined by the new set of knots $\mathcal{K}^{(1)}=\mathcal{K}^{(1)}\left(\xi_{\text {new }}\right)$. In the recurrent process, at the step $s$, we start with a sequence of knots $\mathcal{K}^{(s-1)}$ and search for a new knot $\xi_{\text {new }}$ using (3) with $\mathcal{K}^{(s)}(\xi)=\mathcal{K}^{(s-1)} \cup\{\xi\}$ and the corresponding orthonormal basis of piecewise constant functions $\mathcal{B}^{(s)}(\xi)$.

The algorithm benefits from the locality and orthogonality piecewise constant bases so that each new knot requires a removal only one base function (the constant over interval that includes the new knot) and replaces it by two new functions that remain orthonormal to all the other basis functions from the previous step. The outcome of the zero-order spline decomposition of the FD is shown in Fig. 2, the third column.

\section{Application - efficient analysis of the quarter vehicle model}

The model of a damped harmonic oscillator can be utilized in studies of the durability of vehicle components in the vehicle response to the road profile, see [10] and [7] for further details on the model. The road profile roughness is often quantified using the response of a quarter-vehicle model traveling at a constant velocity through road profiles, see Figure 3. Such a simplification of a physical vehicle cannot be expected 


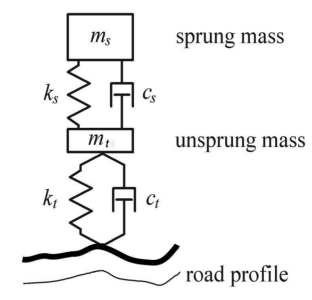

\begin{tabular}{lll}
\hline Parameter Mean & Unit \\
\hline$m_{s}$ & 3400 & $\mathrm{~kg}$ \\
$k_{s}$ & $270000 \mathrm{~N} / \mathrm{m}$ \\
$c_{s}$ & 6000 & $\mathrm{Ns} / \mathrm{m}$ \\
$m_{t}$ & 350 & $\mathrm{~kg}$ \\
$k_{t}$ & 950000 & $\mathrm{~N} / \mathrm{m}$ \\
$c_{t}$ & 300 & $\mathrm{Ns} / \mathrm{m}$ \\
\hline
\end{tabular}

Fig. 3 Quarter vehicle model and examples of its parameters.

to predict loads exactly, but it will highlight the most important road characteristics as far as durability is concerned.

It is desirable to have a model of load environment that is vehicle independent and which may consist of many components, like driving habits, encountered road roughness, hilliness, curve radius, cargo loading, and others. The force acting on the sprung mass $m_{s}$ (total mass of the vehicle) that is randomly distributed around some specific mean value is chosen as the response $y(t)$ from the tire which then is used to compute suitable indexes to classify the severity of road roughness.

In a linear simplification of the problem, the entire system has the following components. The road elevation $R(t)$ that, under constant speed $v$ of the vehicle, linearly drives two damped harmonic oscillators, one representing the tire and the other the wheel suspension system

$$
m_{t} \ddot{u}+c_{t} \dot{u}+k_{t} u=F_{t}, \quad m_{s} \ddot{y}+c_{s} \dot{y}+k_{s} y=F_{s} .
$$

The parameters in the model can be set to mimic heavy vehicle dynamics as, for example, developed in SCANIA, see Fig. 3. They have the following physical interpretation: properties of the tire are described by $k_{t}, c_{t}$, which relate to stiffness and damping of the tire, while properties of the suspension are given by corresponding $k_{s}, c_{s}$. Modeling of true loads acting on components is difficult since tires filter nonlinearly the road profile and the filter parameters depend on very uncertain factors, e.g. tire's pressure, wear, etc. One way to account for the later and simplify the former is to assume that some of the parameters are random and represent properties of the tire in a concrete vehicle on a given trip.

In further simplification, the condition of the trip can be modeled by a Brownian bridge $B$ filtered by a certain kernel $r$. Here the Brownian bridge model reflects small roughness of the road at the beginning and at the end of a trip and an increase of it when the vehicle enters tougher terrain in the middle of the trip. The smoothing kernel $r$ represents road specific properties so that $R(t)=r * d B B(t)$ is the road surface elevation at location $t$. In the literature, many models for the power spectral density $S_{R}$ of road profiles have been proposed, see [1] for a review. Here, the kernel $r$ relates to the $S_{R}$ through the Bochner theorem $r * \tilde{r}(t)=2 \int \cos (\omega t) S_{R}(\omega) d \omega$.

Often one chooses the force acting on the sprung mass as the response $y(t)$ which then is used to compute suitable indexes to classify the severity of road roughness. In the above simplification, this response is linearly driven by the road profile, as it 


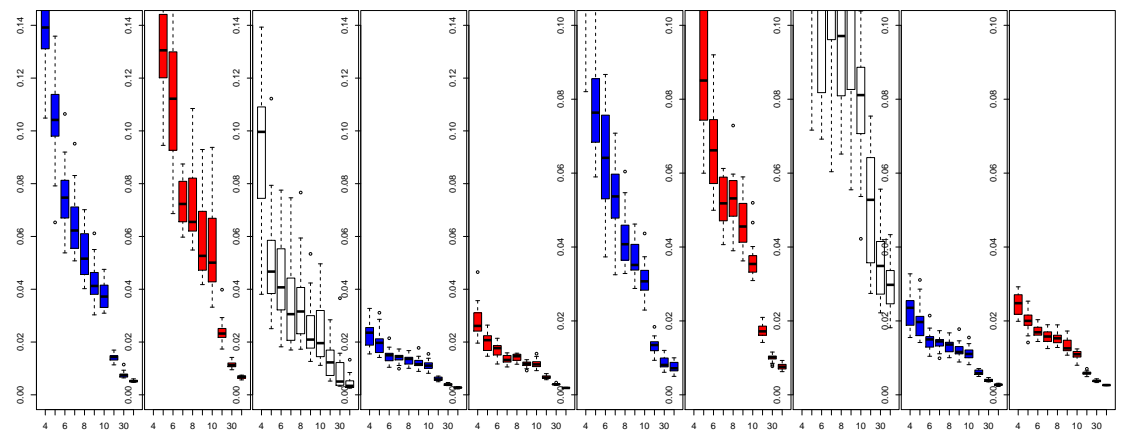

Fig. 4 The boxplots of AMSE's obtained from 20 Monte Carlo simulations for the two models used in Figure 2 as a function of the base size, which ranges from 4 to 40 . For each model, 10 FD were simulated and the orthonormal basis decomposition was run through these FD with an increasing number of basis elements. In each of the two cases grouped in five plots each, in the first and the fourth plot (blue) a new basis is selected anew for each MC sample, while in the second and the fifth (red) a basis is selected only for the original sample and then used for every new MC sample. The first two pictures in each group, correspond to the piecewise constant data-driven basis, the last two to the spline basis, the plots in the middle (black and white) corresponds to the Fourier basis applied to the MC data.

is also the displacement $x(t)$ of the center of the wheel from the road. Their transfer functions, i.e. the Fourier responses to Dirac's delta, are explicit functions of the transfer functions of the two harmonic oscillators

$$
H_{t}(\omega)=-m_{t} \omega^{2}+i \omega c_{t}+k_{t}, \quad H_{s}(\omega)=-m_{s} \omega^{2}+i \omega c_{s}+k_{s} .
$$

To recap, the model is completely defined by the vehicle related parameters: the speed of the vehicle $v$, the mass of the vehicle $m_{s}$, the undamped angular frequencies $\omega_{s}$ and $\omega_{t}$, and the road related parameters, that describe $S_{R}$. All these parameters can be collectively described as $\theta$. Some of these parameters can be considered as random and each observed journey of a vehicle produces a response $y_{i}(t), t \in[0,1]$, with stochastic response driven by samples of Brownian bridge $B_{i}$ and random sample $\theta_{i}$ of the parameters, $i=1, \ldots, n$, where $n$ is the number of trips.

\section{Simulation studies}

We illustrate, through simulations, how using a data-driven orthonormal basis can improve efficiency in representing FD. The setting of Monte Carlo experiment mimics, in a simplified manner, physical systems similar to the quarter vehicle model. The data are not truly sampled from such a model since it would require more extensive study not fitting the format of this note. Instead, we use FD obtained by sampling parameters of the model to which we also inject samples of Brownian bridge

$$
y_{i}(t)=F\left(t ; B_{i}(\cdot), \theta_{i}\right), i=1, \ldots, n .
$$

Ten samples of FD from two such models are presented in Figure 2 (Left).

We performed orthogonal projections to the three ON bases: Fourier, piecewise constant, and the splinets. In Figure 2, we show approximations of the functions 
seen in the left column that uses $N=30$ basis functions. A Monte Carlo study of the dependence of the average mean square error on the number basis elements is shown in Figure 4. Monte Carlo samples of size 10 were drawn from the two models used in Figure 2. For each of these sample approximations with the number of basis elements used increasing from 4 to 50 were evaluated and their average mean square error (AMSE) over all 10 elements of the data evaluated. This procedure has been repeated independently 20 times resulting in 20 AMSE's for each size of the Fourier base used. Boxplots of these data for each model and each the Fourier base size are presented in Figure 4.

\section{Conclusions}

The proposed method of the data-driven orthonormal basis decomposition has been tested in through numerical simulations. The Monte Carlo simulations show clear advantages over the Fourier based method, in particular, when smoothed splines are used. The accuracy is not only exhibited in smaller errors but also in the reduced variability of the error. The improvement is greater, as expected, for the data that shows some local detail. The obtained results suggest that the method may have a great potential to improve the functional analysis of the data coming from the physical systems with random excitation and involving random parameters. This has to be confirmed by further model specific studies.

\section{References}

1. Andrén, P.: Power spectral density approximations of longitudinal road profiles. Int. J. Vehicle Design 40, 2-14 (2006)

2. De Boor, C.: A practical guide to splines, Applied Mathematical Sciences, vol. 27, revised edn. Springer-Verlag New York (2001)

3. Ferraty, F., Vieu, P.: Nonparametric functional data analysis: theory and practice. Springer Science \& Business Media (2006)

4. Guo J., H.J.J.B.Y., Zhang, Z.: Spline-lasso in high-dimensional linear regression. Journal of the American Statistical Association 111(513), 288-297 ((2016))

5. Hastie, T., Tibshirani, R., Friedman, J.: The elements of statistical learning: data mining, inference and prediction, 2 edn. Springer (2009). URL http://wwwstat.stanford.edu/ tibs/ElemStatLearn/

6. Hsing, T., Eubank, R.: Theoretical foundations of functional data analysis, with an introduction to linear operators. John Wiley \& Sons (2015)

7. Johannesson, P., Speckert, M. (eds.): Guide to Load Analysis for Durability in Vehicle Engineering. Wiley, Chichester. (2013)

8. Karhunen, K.: Über lineare Methoden in der Wahrscheinlichkeitsrechnung. Ann. Acad. Sci. Fennicae. Ser. A. 37, 1-79 (1947)

9. Liu, X., Nassar, H., Podgórski, K.: Splinets - efficient orthonormalization of the b-splines. ArXiv abs/1910.07341 (2019)

10. Podgórski, K., Rychlik, I., Wallin, J.: Slepian noise approach for gaussian and Laplace moving average processes. Extremes 18(4), 665-695 (2015). DOI 10.1007/s10687-015-0227-z. URL https://doi.org/10.1007/s10687-015-0227-z

11. Ramsay, J.O.: Functional data analysis. Encyclopedia of Statistical Sciences 4 (2004)

12. Schumaker, L.: Spline functions: basic theory. Cambridge University Press (2007) 